\title{
FATORES PREDISPONENTES À INFILTRAÇÕES PERIFÉRICAS EM CRIANÇAS COM CÂNCER
}

\author{
$\underline{\text { Julia de Paula Silva }}{ }^{1}$; Luciano Marques dos Santos ${ }^{2}$; Katharinne de Jesus Nunes ${ }^{3}$; \\ Cleonara Sousa Gomes e Silva ${ }^{4}$ \\ ${ }^{1}$ Participante do NUDES, Departamento de Saúde, Universidade Estadual de Feira de Santana, \\ e-mail:silvapaulladejulia@gmail.com \\ ${ }^{2}$ Orientador. Departamento de Saúde, Universidade Estadual de Feira de Santana, Pesquisador do NUDES, e-mail: \\ lucmarxenfo@hotmail.com \\ ${ }^{3}$ Bolsista PROBIC/UEFS, Graduanda em Enfermagem, Universidade Estadual de Feira de Santana, \\ e-mail: katharinnenunes@yahoo.com.br \\ ${ }^{4}$ Participante do NUDES, Departamento de Saúde, Universidade Estadual de Feira de Santana, \\ e-mail:cleosilvauefs@gmail.com
}

Palavras-chave: Infiltração; Terapia Intravenosa; Pediatria.

\section{INTRODUÇÃO}

A assistência à saúde é desenvolvida em um sistema complexo, onde predomina a realização de procedimentos e intervenções que podem predispor ao erro e agravar suas consequências, em grau raramente identificado em outras atividades humanas (PEDREIRA, 2006). Dentre esses procedimentos está a Terapia Intravenosa (TIV).

A TIV integra o cotidiano da enfermagem no tratamento dos agravos à saúde, sendo definida como um conjunto de conhecimentos e técnicas que visam à administração de soluções e fármacos no sistema circulatório. Abrange o preparo do paciente, escolha, obtenção e manutenção do acesso venoso, os métodos de preparo e administração de drogas e soluções bem como os cuidados referentes à frequência de troca do cateter curativos, dispositivos de infusão e soluções (PETERLINI; CHAUD; PEDREIRA, 2008).

Em alguns estabelecimentos de saúde os trabalhadores de enfermagem ainda reproduzem um modelo de cuidado sem fundamentação em evidências científicas, expondo a criança hospitalizada à ocorrência de eventos adversos (EA) e a erros relacionados à TIV. EA são definidos como complicações indesejadas decorrentes do cuidado prestado aos pacientes, não atribuídas à evolução natural da doença de base (GALLOTTI, 2004).

A TIV requer a inserção de cateteres intravenosos, periféricos ou centrais, que acessam o sistema venoso, principalmente dos membros superiores. A administração de soluções via intravenosa objetiva repor volume intravascular, corrigir déficits de eletrólitos, administrar fármacos, realizar hemodiálise e fornecer nutrientes ao cliente quando não há outra via disponível (CHRISTOFFEL, 2013).

Esta é uma realidade das unidades de oncologia pediátrica, pois para estabelecer a terapêutica específica para o câncer infantil é necessário a inserção cateter na via venosa periférica ou central e muitos quimioterápicos utilizados no tratamento dessas crianças apresentam características químicas que podem acarretar eventos adversos, a exemplo de infiltração, extravasamento e flebites.

Portanto, o profissional deve estar preparado para preveni-los e caso ocorram identifica-los o mais rápido possível para que sejam evitados grandes danos. A interrupção do evento adverso e a intervenção imediata são condutas praticadas pelos profissionais para minimizar os prejuízos das falhas cometidas (WEGNER; PEDRO, 2008).

Neste estudo dá-se ênfase à infiltração que é definida como o deslocamento do cateter da veia, com consequente saída de solução ou fármaco não vesicante para o espaço extravascular. Pode ser identificada na presença de pele fria ao redor do local de inserção do cateter, edema 
dependente e velocidade de infusão ausente ou lenta (MACHADO; PEDREIRA; CHAUD, 2008).

Em estudo sobre flebite, infiltração e extravasamento em recém-nascidos submetidos à punção intravenosa periférica, a análise da variável de infiltração revelou que 79,2\% nos recém- nascidos avaliados apresentaram infiltração (GOMES et al., 2011).

No entanto, além de saber identificar uma infiltração é importante que os profissionais de enfermagem saibam quais são as crianças que estão mais propensas a ter esse tipo de complicação através do reconhecimento de quais são os fatores de risco relacionados a sua ocorrência, porém os estudos sobre o tema ainda é escasso, principalmente quando relacionado especificamente a oncologia.

Diante disto, este estudo teve como objeto de investigação os fatores associados a ocorrência de infiltrações intravenosas em crianças com câncer.

\section{MÉTODOS}

Estudo transversal, realizado crianças/adolescentes de idade entre 29 dias e 16 anos, com necessidade de TIV por via periférica, hospitalizadas em unidaade de clínica oncológica de um hospital pediátrico de Feira de Santana na Bahia. Os dados foram coletados no período de fevereiro de 2015 a novembro de 2016, através de um formulário e foram processados no Statistical Package for Social Sciences versão 22.0. Foram calculadas as frequências absolutas e relativas de cada variável. $\mathrm{Na}$ análise bivariada foi calculado a razão de prevalência e o intervalo de confiança. Foi utilizado o Qui-quadrado de Pearson com nível de significância de 5\% (p<0,05) e o Exato de Fisher. Na análise multivariada foi realizada a Regressão de Poison. Este estudo respeitou a Resolução 466/12 do conselho Nacional de Saúde, sendo aprovado pelo Comitê de Ética em Pesquisa da Universidade Estadual de Feira de Santana.

\section{RESULTADOS}

Neste estudo foram observados 309 dispositivos intravenosos periféricos inseridos para realização da TIV, dentre estes 38 apresentara infiltração e foi observada uma taxa de complicação de $12,3 \%$. As infiltrações identificadas foram avaliadas e classificadas em graus conforme a escala da INS (2016), sendo mais prevalente as de Grau 1 (60,0\%).

Ao analisar as variáveis relacionadas a crianças/adolescentes foi possível observar que $34,4 \%$ apresentavam- se na idade pré-escolar (36 a 83 meses)e idade média de 89,13 meses e idade mínima de 9 meses e máxima de 194, 59,4\% do sexo feminino e 59,9\% pardas.

Segundo as condições clínicas, 78,1\% das crianças estavam eutróficas, 84,4\% não tinham histórico de prematuridade, $96,9 \%$ sem edema e $68,7 \%$ não estavam hiperativas no momento de realização da CIP, com tempo de hospitalização inferior a sete dias e o sistema orgânico mais acometido, segundo diagnóstico médico, foi o hematológico $(59,4 \%)$.

Para as variáveis relacionadas à TIV prévia utilizada e mostra que $62,5 \%$ das crianças possuíam histórico de dificuldade para inserção do cateter, $81,2 \%$ não foram submetidas à TIV prévia prolongada, 90,6\% já haviam usado CIP, 87, 5\% apresentaram antecedentes de complicações, 57,1 \% não apresentaram antecedente de flebite, 85,7\% apresentaram histórico de infiltração, 96,4\% não apresentaram extravasamento e 64,3\% apresentaram obstrução.

Das variáveis relacionadas à CIP atual observa-se que 56,3\% das cateterizações foram realizadas no MSD, 71,9\% das veias eram visíveis, $68,8 \%$ palpáveis, $71,9 \%$ retilíneas, $84,4 \%$ superficiais. O tipo de cateter mais utilizado foi o de poliuretano (65,6\%), 24 Gauges (46,9\%), em $93,8 \%$ foi utilizado o método de punção direto, estabilização feita em $84,4 \%$ das crianças e em $78,1 \%$ a cobertura foi feita com a cobertura estéril e micropore.

Ao analisar as variáveis relacionadas à TIV atual utilizada pelas crianças encontrou-se que $90,6 \%$ delas fizeram uso de medicamentos irritantes, $46,9 \%$ de vesicantes, $68,8 \%$ utilizaram 
soluções vesicantes, 40,6\% tiveram suas drogas e soluções administradas através de dois métodos associados (Sistema eletrônico de infusão + bureta). A infusão da TIV ocorreu em $76,7 \%$ delas de forma intermitente e contínua e a maioria utilizou fármaco, solução e eletrólito $(43,8 \%)$, das crianças utilizaram a CIP por menos de 72 horas, utilizaram um medicamento irritante $(65,5 \%)$, um medicamento vesicantes $(100 \%)$, um medicamento não irritante/vesicante $(72,7 \%)$, uma ou duas soluções vesicantes ( 40,9\%) e uma ou duas soluções não irritantes/vesicantes. As soluções mais utilizadas foram os eletrólitos $17,8 \%$ e antieméticos $17 \%$.

$\mathrm{Na}$ analise bivariada as variáveis demográficas e clínicas das crianças/adolescentes e a ocorrência de infiltração, encontrou-se associação estatística entre os grupos com e sem infiltração com a condição nutricional da criança $(\mathrm{RP}=2,3 ; \mathrm{p}=0,028)$ e tempo de hospitalização $(\mathrm{RP}=5,6 ; \mathrm{p}=0,000)$.

$\mathrm{Na}$ análise das associações entre as variáveis relacionadas à TIV prévia e a ocorrência de infiltração, foi possível observar associação estatística entre as crianças que apresentaram e não apresentaram infiltração para histórico de dificuldade de $\mathrm{CIP}(\mathrm{RP}=2,9 ; \mathrm{p}=0,000)$, para aquelas que passaram por TIV periférica prolongada $(\mathrm{RP}=5,6 ; \mathrm{p}=0,000)$ e para aquelas com antecedente de complicações ( $\mathrm{RP}=3,6 ; \mathrm{p}=0,009)$.

Com a associação entre as variáveis relacionadas a CIP atual, foi possível notar associação entre visibilidade da veia $(\mathrm{RP}=2,3 ; \mathrm{p}=0,015)$ e a estabilização do cateter $(\mathrm{RP}=12,6 ; \mathrm{p}=0,000)$.

Ao analisar as associações entre as variáveis relacionadas à TIV atual utilizada foi possível observar associação entre as crianças/adolescentes que fizeram uso de medicamentos irritantes $(\mathrm{RP}=2,9 ; \mathrm{p}=0014)$, as que fizeram uso de medicamentos vesicantes $(\mathrm{RP}=0,5$; $\mathrm{p}=0,030)$, uso de medicamentos não irritante e não vesicante $(\mathrm{RP}=2,4 ; \mathrm{p}=0,005)$, uso de soluções vesicantes $(\mathrm{RP}=4,1 ; \mathrm{p}=0,000)$, uso de soluções não irritantes e não vesicantes ( $R P=2,7 ; p=0,007)$, infusão contínua da TIV $(R P=0,3 ; p=0,005)$, e tempo de utilização da CIP superior ou igual a 72 horas $(\mathrm{RP}=2,6 ; \mathrm{p}=0,026)$.

Com a realização da analise multivariada às variáveis relacionadas à TIV prévia, observadas na Tabela 10, confirmou-se que crianças e adolescentes que possuem histórico de dificuldade da CIP ( $R P=2,3 ; p=0,006)$, que utilizaram TIV periférica prolongada $(\mathrm{RP}=5,1 ; \mathrm{p}=0,000) \mathrm{e}$ com antecedentes de complicação $(\mathrm{RP}=3,9 ; \mathrm{p}=0,019)$ estão mais propensas à ocorrência de infiltração.

Com relação às variáveis relacionadas à CIP atual, confirmou-se que as crianças/adolescentes que possuem veias não visíveis $(\mathrm{RP}=2,4 ; \mathrm{p}=0,008)$ e não palpáveis $(\mathrm{RP}=1,9 ; \mathrm{p}=0,035)$ estão mais propensos a infiltração.

Das variáveis relacionadas à TIV atual, confirmou-se que as crianças/adolescentes que utilizaram medicamentos não irritantes/vesicantes $(\mathrm{RP}=1,8 ; \mathrm{p}=0,054)$ e soluções vesicantes $(\mathrm{RP}=3,6 ; \mathrm{p}=0,000)$ estão mais propensas a infiltração.

\section{CONCLUSÕES}

Com a realização deste estudo foi possível encontrar fatores predisponentes a ocorrência de infiltração em crianças/adolescentes submetidos a TIV periférica, em unidade oncológica. Diante disso foi confirmada a hipótese de que existem fatores predisponentes a ocorrência de infiltração quando são analisadas as características da criança, da CIP, da TIV prévia e da atual.

A regressão de Poisson confirmou a associação entre as variáveis correspondentes ao histórico de dificuldade da CIP ( $\mathrm{RP}=2,3 ; \mathrm{p}=0,000)$, TIV prévia prolongada $(\mathrm{RP}=5,1$; $\mathrm{p}=0,000)$, antecedentes de complicação ( $\mathrm{RP}=2,9 ; \mathrm{p}=0,009)$, visibilidade ( $\mathrm{RP}=2,3 ; \mathrm{p}=0,015)$, palpabilidade $(\mathrm{RP}=1,9 ; \mathrm{p}=0,035)$, uso de medicamento não irritante/vesicante ( $\mathrm{RP}=1,8$; $\mathrm{p}=0,054)$, uso de solução vesicante $(\mathrm{RP}=3,6 ; \mathrm{p}=0,000)$ e a ocorrência de infiltração. 
Este estudo oferece contribuições teóricas já que existem poucos estudos a cerca dos fatores associados à ocorrência de infiltrações e esse cenário se torna ainda mais escasso ao levar em consideração a população pediátrica com diagnóstico de câncer.

No campo de prática esse estudo pode auxiliar o profissional na identificação de crianças que apresentem os fatores apresentados nesta pesquisa, fazendo com que o mesmo possa priorizar essa crianças/adolescente para uma avaliação mais rigorosa e pensar formas de prevenir e tratar caso elas venham a acontecer.

O presente estudo apresenta algumas limitações, pois para a análise bivariada e multivariada foram avaliados os dispositivos intravenosos ao invés de crianças, por se tratar de um estudo transversal não é possível generalizar seus resultados para toda população e por ter utilizado os dados do internamento e ambulatório.

\section{REFERÊNCIAS}

CHRISTOFFEL, M. 2013.Terapia intravenosa: práticas de enfermagem para uma assistência de qualidade. Programa de Aprimoramento Profissional - Proficiência. Conselho Federal de

| Enfermagem. GALLOTTI, -R. M. D. 2004. Eventos adversos - o que são?. Revista

Associação Medica Brasil.50(2):109-26. Disponível em: < http://www.scielo.br/pdf/ramb/v50n2/20754.pdf > Acesso em: 9 de julho de 2017.

GOMES, A. C. R et al. 2011. Avaliação da ocorrência de flebite, infiltração e extravasamento em neonatos submetidos à terapia intravenosa. Esc Anna Nery. 15 (3): 472479.

PEDREIRA, M. L.G. Errar é humano: estratégias para a busca da segurança do paciente. In: HARADA, M. J. C. S.; PEDREIRA, M. L. G.; PETERLINI, M. A. S.; PEREIRA, S.G. $O$ erro humano e a segurança do paciente. São Paulo, Atheneu, 1-18.

PETERLINI, M. A. S.; CHAUD, M. N.; PEDREIRA, L. G. P. 2008. Órfãos de terapiamedicamentosa: a administração de medicamentos por via intravenosa em criançashospitalizadas. Rev. Latino-Am. Enferm,- 11(1) 88-95. .

MACHADO, A. F.; PEDREIRA, M. L. G.; CHAUD, M. N. 2008. Eventos adversos relacionados ao uso de cateteres intravenosos periféricos em crianças de acordo com tipos de curativos. Rev. Latino-Am. Enfermagem.16(3):362-367. 